\title{
e-Phaïstos
}

e-Phaïstos

Revue d'histoire des techniques / Journal of the history

of technology

VIII-1 | 2020

Écomusée : une expansion internationale

\section{Recherche anthropologique et écomusées : une utopie assumée}

Anthropological Research and Ecomuseums : Accepting Utopia

\section{Thierry Bonnot}

\section{(2) OpenEdition}

Journals

Édition électronique

URL : http://journals.openedition.org/ephaistos/7424

DOI : 10.4000/ephaistos.7424

ISSN : 2552-0741

Éditeur

IHMC - Institut d'histoire moderne et contemporaine (UMR 8066)

Référence électronique

Thierry Bonnot, « Recherche anthropologique et écomusées : une utopie assumée », e-Phaïstos [En

ligne], VIII-1 | 2020, mis en ligne le 29 avril 2020, consulté le 06 mars 2021. URL : http://

journals.openedition.org/ephaistos/7424 ; DOI : https://doi.org/10.4000/ephaistos.7424

Ce document a été généré automatiquement le 6 mars 2021.

Tous droits réservés 


\title{
Recherche anthropologique et écomusées : une utopie assumée
}

\author{
Anthropological Research and Ecomuseums : Accepting Utopia
}

\section{Thierry Bonnot}

1 Ce texte n'émane pas d'un spécialiste des écomusées, dans le sens où l'écomuséologie n'est pas mon objet de recherche, mais il est l'œuvre d'un praticien des écomusées, comme public et comme chercheur, qui souhaite ici partager un ensemble de réflexions. Je suis surtout praticien d'un écomusée, celui du Creusot-Montceau, auquel je dois d'être là : j'y ai mené mes premières recherches, j'y ai fait ma thèse, j'en ai été salarié huit ans et continue de travailler sur son territoire, le plus souvent en coordination avec l'équipe actuelle. Ce qui suit sera donc fortement inspiré par cette vingtaine d'années de collaboration et le lecteur voudra bien excuser ce tropisme peutêtre un peu trop marqué.

2 En fonction de ces limites, je ne me permettrai ni de revenir sur l'histoire des écomusées, ni de tenter un tour de France - encore moins un tour du monde ! - des écomusées. Il s'agit pour moi d'amener des idées au centre des débats, de lancer des pistes de réflexion autour d'un axe principal : le rôle et la pratique de la recherche dans le travail de l'écomusée, pour m'en tenir à ce que je connais le mieux. J'évoquerai les liens privilégiés de l'ethnologie et de l'anthropologie avec les écomusées, liens basés sur la notion de terrain et celle de territoire. Je tenterai de montrer comment la recherche de terrain n'est pas seulement une collecte de données ou d'objets, mais participe pleinement à l'activité des écomusées telle que l'avaient définie ses fondateurs. Rien de bien novateur dans tout cela, car je reste persuadé que dans le domaine qui nous intéresse l'innovation consiste à se réapproprier les idées d'origine.

3 Un point de vocabulaire préalable: lorsque j'emploierai ici le terme d'écomusée, il faudra aussi entendre "musée de société». Ce n'est pas seulement une facilité de langage, mais le constat que la plupart du temps, il n'est pas pertinent de distinguer les deux, dans leur ambition, dans leur programme comme dans leurs pratiques. 


\section{L'écomusée, utopie ou aporie?}

4 Dans l'abondante littérature consacrée aux écomusées, à leur projet intellectuel et à leur histoire, la notion d'utopie est de celles qui reviennent le plus fréquemment. Ainsi, un exemple parmi d'autres ${ }^{1}$, Philippe Mairot écrit-il en 1992 :

«La plus radicale et la plus difficile de ses innovations, c'est, me semble-t-il, la place que l'écomusée s'assigne au sein de la société. Il veut être ce lieu central de la vie communautaire, forum et non plus temple, lieu de dialogue avec l'altérité, de confrontation avec son passé où la société peut décider de son développement en toute connaissance de ses déterminations géographiques, historiques, sociales, culturelles et économiques. Ce musée est une utopie. Il ne veut rien moins qu'être ce cristal où se reflèterait la réalité vraie $[\ldots]^{2} »$.

$5 \quad$ Pour la plupart des auteurs, il s'agit de démontrer que les idées de base du mouvement écomuséal étaient vouées presque par définition à l'inaccomplissement, comme si elles restaient un idéal que les contradictions et les rêves délirants des années 1960 et 1970 rendaient inéluctablement inaccessible. Plusieurs facteurs expliqueraient ainsi que l'ambition théorique originelle était inapplicable en pratique. Le paradoxe terminologique fait partie du problème: alors que ce qui faisait l'originalité, voire la radicalité du projet était la distinction marquée entre écomusée et musée, la dénomination choisie a brouillé les pistes. Un écomusée n'est pas un musée, mais il contient dans son nom ce dont il veut se distinguer! Si l'on admet que l'écomusée a été en partie pensé contre le modèle classique du musée, admettons aussi que le choix de ce terme était, sinon ambigu, du moins potentiellement ouvert à " mille pratiques ${ }^{3}$.

Mais il me semble que, parfois, les auteurs confondent utopie et aporie. En l'occurrence, l'idée d'un musée sans collection serait une aporie, soit une difficulté rationnelle insurmontable, une contradiction sans issue, une impasse logique. Ce qui induit l'échec inévitable du projet écomuséal. Mais l'utopie, quant à elle, n'est pas synonyme d'échec. La dérive du sens de ce mot l'a fait assimiler à une chimère, quelque chose qui ne se réalisera jamais. C'est ainsi qu'il est utilisé pour disqualifier les projets politiques supposés irréalistes, c'est-à-dire non conformes au discours dominant. Pourtant l'origine du mot utopie en donne une vision plus positive. Utopia est le pays imaginaire inventé par Thomas More en 1516, république idéale où vit heureux un peuple gouverné idéalement ${ }^{4}$. Il ne s'agit plus alors de qualifier d'utopique un projet aberrant d'un point de vue logique, mais d'envisager l'utopie comme un objectif, sans doute lointain mais dont il faut entretenir la petite flamme pour qu'elle continue à donner sens à nos actions. Vue sous cet angle, en quoi consiste l'utopie écomuséale?

7 Nous pouvons lancer quelques pistes, à partir des définitions données par ceux qui sont considérés comme les inventeurs du concept. D’abord, Georges-Henri Rivière, et sa fameuse métaphore du miroir :

«Un écomusée est un instrument qu'un pouvoir et une population conçoivent, fabriquent et exploitent ensemble. Ce pouvoir, avec les experts, les facilités, les ressources qu'il fournit. Cette population, selon ses aspirations, ses savoirs, ses facultés d'approche. Un miroir 
où cette population se regarde, pour s'y reconnaitre, où elle recherche l'explication du territoire auquel elle est attachée, jointe à celle des populations qui l'ont précédée, dans la discontinuité ou la continuité des générations. Un miroir que cette population tend à ses hôtes, pour s'en faire mieux comprendre, dans le respect de son travail, de ses comportements, de son intimité $»^{5}$.

Hugues de Varine, ensuite:

\begin{abstract}
«Le musée "normal" est un bâtiment, une collection, des publics / L'écomusée est un territoire, des patrimoines, une communauté. Le musée normal, c'est de la culture "hors-sol". L'écomusée, c'est un musée enraciné dans la culture vivante des habitants $»^{6}$.
\end{abstract}

Retenons donc que le projet écomuséal repose sur un travail collectif, associant pouvoir et population; et un travail enraciné dans une culture vivante. Ce sur quoi je veux insister, c'est que ce travail collectif et cet enracinement passent par la recherche, principalement par la recherche en sciences sociales et que l'utopie, au sens le plus fécond du terme, est peut-être l'idéal d'une recherche dialogique, non surplombante, pluridisciplinaire et ouverte, ce qui n'a rien d'une aporie.

\title{
Ethnologie, anthropologie et écomusées
}

10 La discipline motrice des écomusées, même s'ils se revendiquent à juste titre pluridisciplinaire, c'est l'ethnologie. C'est un postulat évidemment discutable, car tout dépend de ce que l'on rassemble sous cette bannière, mais admettons toutefois ces arguments généalogiques: en France, les écomusées et musées de société sont les héritiers du Musée National des Arts et Traditions Populaires - dont certains ont même conservé l'intitulé - et leur développement ressemble à un essaimage sur l'ensemble du territoire de musées d'ethnologie de la France. À partir de 1980, c'est la Mission du Patrimoine Ethnologique du Ministère de la Culture et son réseau d'ethnologues régionaux qui ont été les référents institutionnels et, pour une part importante, les financeurs des écomusées et des musées de société7.

11 Au-delà de ce lien théorique et institutionnel, la présence de la discipline ethnologique sur le terrain passe par l'action de chercheurs, dont le rôle et l'image reflètent le flou relatif qui entoure la définition de l'ethnologie de la France. Issues de mon expérience personnelle sur le territoire de l'écomusée du Creusot-Montceau, deux anecdotes nous permettront de cerner partiellement la question. D'abord cette remarque d'un conseiller régional de Bourgogne venu visiter le chantier-école de réhabilitation de la briqueterie Vairet-Baudot (Ciry-le-Noble, Saône-et-Loire) ${ }^{8}$. Il s'était montré intéressé par la maçonnerie, la charpente, la mécanique et par les résultats probants en matière d'insertion sociale. J'avais été présenté comme «l'ethnologue chargé du projet pour l'écomusée ", délégué sur place par la direction. Au milieu de la visite, le conseiller régional s'est tourné vers moi en demandant: «Mais l'ethnologue, qu'est-ce qu'il fait là-dedans ? » Le sous-entendu était limpide : pourquoi de l'ethnologie dans un chantier de réhabilitation? Un historien de l'industrie, un architecte ou un archiviste n'auraient-ils pas été mieux à leur place, fournissant à l'encadrement professionnel les données nécessaires sur l'historique du bâti? 

Portet, ethnologue régional à la DRAC Bourgogne dans les années 1990-2000. Je lui demandais pourquoi c'était à l'ethnologue ${ }^{9}$ de s'occuper de l'histoire industrielle, de répertorier les témoignages et les savoir-faire, de collecter et étudier tous ces objets du quotidien. Il m'a répondu : « Puisque personne ne s'y intéresse, c'est à l'ethnologie de le faire ». Pour le dire autrement: ce que les disciplines spécialisées dans l'histoire, le social, la technique, laissent de côté, c'est à l'ethnologie de s'en préoccuper. Cette discipline, née de l'aventure coloniale, vouée initialement à l'étude des peuples exotiques, lorsqu'elle se tourne vers son propre milieu, ramasserait ainsi les miettes des nobles disciplines sœurs, du moins se contente-t-elle de ce que les autres ont négligé. De façon plus constructive, disons que l'ethnologie trouve sa pertinence dans l'étude de phénomènes plus ou moins marginaux, mais dont l'importance s'avère décisive pour les grands sujets des sciences humaines et sociales. Ce que nous disent cette question et cette réponse indirecte peut nous permettre de réfléchir à l'articulation entre le projet écomuséal et la recherche en sciences sociales. moins assumée du structuralisme, est farouchement jalouse de ses particularismes ${ }^{10}$, une anthropologie critique et descriptive s'efforce de renouveler ses méthodes et de s'ouvrir aux autres disciplines des sciences humaines et sociales. Celle-ci se revendique davantage comme anthropologie au sens où l'a définie Pierre Bourdieu dans une intervention tardive :

«[...] je ne puis néanmoins m'empêcher de souhaiter voir l'unité des sciences de l'homme s'affirmer sous le drapeau d'une Anthropologie désignant à la fois, dans toutes les langues du monde, ce que l'on entend aujourd'hui par ethnologie et par sociologie $»^{11}$.

Cet élargissement épistémologique tend à s'extraire de la distinction hiérarchique inventée par Lévi-Strauss - l'ethnographe travaille sur le terrain, l'ethnologue analyse les données en cabinet, l'anthropologue compare et synthétise - et, pour ce qui concerne les écomusées, rend plus poreuse la frontière entre ethnologie du lointain et ethnologie de la France. L'anthropologie, qu'elle s'intéresse aux Bambaras ou aux habitants du Charolais bourguignon, est une science de l'homme, caractérisée par l'enquête de terrain mais empruntant méthodologiquement à l'histoire, à la sociologie et à l'archéologie. Ce sont d'ailleurs des archéologues qui ont le mieux, à mon sens, posé récemment le cadre de ce qui pourrait modifier les découpages disciplinaires les plus anciens. Florence Journot et Gilles Bellan, dans les toutes dernières phrases de leur conclusion de l'ouvrage Archéologie de la France moderne et contemporaine, l'affirment avec force :

« Il y aurait profit à organiser l'échange entre acteur des sciences humaines. La communication entre spécialistes de l'humain devrait se prolonger au-delà des juxtapositions de communications lors des colloques. [Il faudrait] construire une interdisciplinarité digne de ce nom, d'une belle ambition scientifique : et si cet échange raisonné s'appelait "anthropologie"?»".

Que cette proposition émane d'archéologues évite de les accuser d'un quelconque impérialisme académique au profit de l'anthropologie. Il s'agit bien de construire un 
espace interdisciplinaire dont l'objet est l'homme en société, et, pour ce faire, d'emprunter les méthodes et les outils de l'ensemble des disciplines traditionnellement fractionnées. Ainsi définie, la recherche anthropologique est peut-être plus attentive que d'autres à certains faits car basée sur une méthode empirique, l'enquête de terrain qui «fait feu de tout bois [et] s'appuie sur tous les modes de recueil de données possibles $»^{13}$. C'est bien ainsi que doit être envisagé le travail de recherche d'un écomusée, parfois en rupture avec les cadres disciplinaires les plus rigides, mais en phase avec une approche éclectique de ce que l'on appelle le terrain.

\section{Le terrain et le territoire}

L'une des raisons de l'association logique entre les écomusées et l'anthropologie est l'assimilation d'un territoire au terrain de l'enquête. L'anthropologie est par excellence la discipline de l'enquête de terrain ; l'écomusée est par excellence l'institution définie par un territoire. L'articulation paraît donc évidente, bien qu'il s'agisse de deux notions différentes puisque pour l'anthropologue, le terrain n'est pas seulement une réalité topographique ou administrative. Le terrain anthropologique est d'abord une expérience de longue durée, un travail qui doit tenir compte des enjeux mouvants et des rapports de force évolutifs. Selon Jean Bazin, l'anthropologue n'étudie pas des sociétés car une société n'est pas une chose que nous pouvons observer; nous n'observons jamais que des situations. Nous partageons celles-ci avec les individus sociaux que nous étudions, ceux dont « le monde n'est pas le mien » :

\footnotetext{
«Dans la mesure où leur monde n'est pas le mien, il est pour moi objet de savoir. Mais c'est un objet qui m'est donné dans une situation qu'eux et moi partageons. Cette tension entre co-présence et distance définit ce qu'on appelle (par opposition au laboratoire ou au cabinet de travail) "le terrain" ${ }^{14}$.
}

17 Le terrain, c'est donc la rencontre entre un observateur et des individus dans le cadre de situations, où le premier cherche à comprendre ce que font les seconds. Dans une perspective très proche, les habitants du territoire de l'écomusée, qui constituent aussi son public ${ }^{15}$, sont coproducteur de savoirs, en tout cas, ils ne sauraient être considérés ni comme simple consommateurs culturels, ni comme purs sujets d'observation. C'est l'une des plus hautes exigences - elle aussi utopiques? - du travail de l'écomusée : produire du savoir en faisant participer le public à cette production. D'où l'idée fondatrice, assez neuve au début des années 1970, de la mise en place de "Comités des usagers ", au Creusot et ailleurs. On entendait le terme usager au sens large, qui est très différent de celui de public, encore plus éloigné de ceux de consommateur ou client. Il s'agissait de faire participer la population du territoire communautaire au projet écomuséal, en impliquant des responsables d'associations, des érudits locaux, des anciens ouvriers des entreprises locales, des syndicalistes, etc. Les historiens de l'écomusée sont assez circonspects sur l'efficacité de ces comités. Frédéric Poulard note même que :

«dans l'ensemble des écomusées [...] la participation des représentants de la population ne va pas de soi car un flou persiste 
quant à la place qui leur est accordée. En effet, dans une majorité d'écomusées, le comité des usagers ne s'est jamais réuni $»^{16}$. années 1970 et 1980 n'existent plus. Mais l'écomusée a changé de statut en 2012 ; il n'est plus associatif, devenu un service de la Communauté Urbaine Le Creusot-Montceau. Ses missions sont désormais étroitement associées aux collections (récolement, expositions, acquisitions) et l'animation du réseau des antennes n'est plus, pour l'heure, une priorité.

\section{Une recherche faite de rencontres}

L'idée de rencontre ou de co-présence évoquée par Jean Bazin est capitale en anthropologie, qui a pu être définie comme la discipline de la rencontre par excellence :

«L'anthropologie n'est pas un discours philosophique détaché du monde de l'expérience : elle est par excellence la discipline de la rencontre avec les différentes formes de vie, que celles-ci appartiennent aux localités où travaille les anthropologues ou celles auxquelles ils appartiennent eux-mêmes $»^{17}$.

Pour Bernard Traimond, cette particularité de la discipline participe de la définition de son matériau :
«Les sources de l'anthropologue n'expriment pas une réalité qui serait directement donnée par un locuteur informé mais résultent de relations par lesquelles chacun, enquêteur et indigène, apporte son propre savoir, né dans la chaleur d'une rencontre singulière» ( $\mathrm{p}$. $133)^{18}$.

Il s'agit de rencontrer l'autre, pour le comprendre, de se trouver en présence de personnes partageant la même temporalité et participant au moins ponctuellement de la même situation. L'enquête anthropologique n'est pas une simple opération de collecte de données sur un terrain-territoire, que ces données soient des discours, des textes, des mythes, ou des objets matériels.

\footnotetext{
« Nous ne pouvons plus nous en tenir à l'idée que l'enquête est une collecte d'informations éparses à disposition du chercheur comme des champignons dans une forêt » écrit Alban Bensa ${ }^{19}$. Dit autrement, par Jean-Pierre Olivier de Sardan, les données de l'anthropologue "ne sont pas des "morceaux de réel" cueillis et conservés tels quels par le chercheur (illusion positiviste), pas plus qu'elles ne sont de pures constructions de son esprit ou de sa sensibilité (illusion subjectiviste). Les données sont la transformation en traces objectivées de "morceaux de réel" tels qu'ils ont été sélectionnés et perçus par le chercheur $»^{20}$.
}

Les informations collectées par entretien résultent d'une interaction dans laquelle le chercheur joue un rôle décisif et dans le cadre d'un écomusée ou d'un musée en 
général, les objets jouent un rôle et sont à considérer dans le cadre de cette interaction. Ce choix méthodologique et épistémologique amène à utiliser des sources variées, parfois hétérogènes. Pour résumer, l'anthropologie des écomusées est une anthropologie critique, historique et réflexive, qui assume sa posture de science humaine dont les résultats ne sont pas assimilables à ceux des sciences dures. Les objets collectés le sont dans le cadre de situations - d'où l'attention portée à l'enquête telle qu'elle se fait - dont ils ne doivent pas être radicalement détachés afin de saisir toute la richesse de leur histoire et de leur statut social.

Le rôle de la recherche dans un écomusée ne consiste donc pas seulement à l'accumulation de connaissances destinées à alimenter les collections et les actions culturelles. Elle est une démarche collective, en dialogue avec les habitants, les antennes, les réseaux locaux et extérieurs. La succession de rencontres plus ou moins articulées et interdépendantes qui construit le terrain de recherche est faite d'interactions entre le chercheur et des habitants, des témoins plus ou moins dotés d'expertise, mais également des rencontres avec les choses : paysages, bâtiments, objets mobiliers, archives écrites, images, matières minérales, animaux, végétaux, etc. Pour reprendre une terminologie latourienne ou descolienne ${ }^{21}$, les rencontres se jouent aussi bien avec des humains qu'avec des non-humains. Ces derniers ne sont pas seulement des matériaux d'enquête au sens de données qu'il s'agit de collecter. Ils sont les matériaux qui font l'enquête; ils en sont à la fois le substrat, le carburant et les personnages principaux.

\section{Patrimoine ou patrimoines ?}

Admettre ces principes nous amène à remettre en question quelques notions profondément ancrées dans le discours des acteurs. Au cours de l'été 2016, à propos de projets de recherche autour d'un site de patrimoine industriel, j'ai reçu ce message d'un salarié de l'écomusée du Creusot Montceau concernant des habitants d'un village : "Mon expérience ne me permet pas de dire que j'ai ressenti un engouement fort pour le Patrimoine de la part des habitants ». Cette remarque un peu désabusée s'appuie sur une vision positiviste du Patrimoine avec P majuscule tout en établissant un constat d'échec par rapport à l'intérêt d'une population pour ce Patrimoine, ainsi officiellement défini. Pourtant, outre que l'attachement à un monument apparaît surtout au moment où ledit monument est en péril, l'engouement de la population ne serait-il pas plus fort si elle était véritablement impliquée dans la construction des patrimoines et pas seulement dans la réception et l'admiration d'un Patrimoine défini d'en-haut?

De nombreux chercheurs en sciences sociales se sont efforcés de déconstruire la notion de patrimoine, d'apporter un point de vue critique en essayant de maintenir sa légitimité sociale et de concilier perspective scientifique et vision de sens commun ${ }^{22}$. Ils ont bien montré que les patrimoines sont des mémoires vivantes, des répertoires culturels partagés, construits collectivement et transmis, des systèmes de références sociaux et mémoriels. Le Patrimoine avec un grand $\mathrm{P}$, outre son image élitiste, est perçu à juste titre comme poussiéreux, renvoyant exclusivement à un passé révolu et si possible prestigieux, reflétant l'âge d'or d'un territoire: vision du patrimoine sans doute très "franco-française ». Pour sortir de cette perspective étriquée, l'ouverture des écomusées au contemporain s'avère cruciale. S'intéressant aux problématiques 
actuelles traitées par l'anthropologie et la sociologie, de nombreux écomusées s'assument comme acteurs de la vie contemporaine, interrogeant les patrimoines comme catégorie dans leur dynamique, qui implique connaissance de soi et ouverture à l'autre $^{23}$. Nous retrouvons ici le miroir de Georges-Henri Rivière. Les écomusées engagés, au sens politique du terme, dans le contemporain, nous parlent de la ville, des migrations, du rapport à la nature, des paysages, du genre, etc. Sur les thématiques des techniques et de la culture matérielle, les travaux récents sur la question des déchets, de la virtualité, du rapport aux objets et ses mutations contemporaines font partie des questions que pourrait s'approprier l'écomusée ${ }^{24}$. L'articulation bien pensée entre écomusée et recherche anthropologique, si l'on s'extrait des schémas anciens, pourrait générer une refondation profonde et stimulante du projet écomuséal et de son ancrage dans la modernité.

\section{Retrouver le goût de l'utopie contre le consumérisme culturel}

La recherche en sciences sociales, menée sur la base d'une anthropologie ouverte sur les outils et concepts d'autres disciplines, ancrée dans l'enquête de terrain, doit être le moteur de l'action écomuséale. Pas seulement pour augmenter les collections, remplir les vitrines et multiplier les publications des écomusées devenus, bon gré mal gré, des musées, mais pour :

$1^{\circ}$ mieux connaître le territoire d'action et ses habitants et engager une dynamique collective adaptée ;

$2^{\circ}$ associer la population à l'action de recherche et transformer les informateurs en coauteurs ;

$3^{\circ}$ susciter ainsi un mouvement de développement local ancré dans une vision des patrimoines comme processus articulant passé, présent et avenir.

La recherche participe du développement social et culturel auquel aspirent, en théorie, les écomusées. Ce développement n'est pas celui de l'économie industrielle néolibérale - où développement $=$ croissance $=$ PIB $=$ productivité, etc., - mais un développement ouvert sur un véritable projet de société.

Car «il faut aussi se demander quelle place la société fait à ses musées, si elle les considère comme de véritables outils de développement, ou bien si elle les cantonne dans un rôle de consommation culturelle, au profit des élites du territoire, des groupes scolaires encadrés et des touristes ${ }^{25}$.

L'anthropologie a un rôle à jouer dans ce type de développement non exclusivement économique, par son rôle critique et par sa capacité à dialoguer avec le public et les acteurs sociaux locaux, à faire dialoguer ces derniers avec des acteurs non locaux. La connaissance en profondeur du terrain, fruit d'une implication de longue durée, permet l'existence d'un réseau qu'il faut encourager, dynamiser, soutenir ; l'écomusée doit susciter l'engagement patrimonial et fédérer les acteurs. Par la description anthropologique de situations, il s'agit de faire comprendre ce qui se passe ici et de faire comprendre que ce n'est pas sans lien avec ce qui se passe ailleurs. Nous suivons sur ce point Marc Augé :

«C'est bien du même enjeu qu'il s'agit toujours: comment approfondir suffisamment la vérité d'un moment ou d'un lieu pour la 
rendre perceptible à chacun et à tous, dépasser l'idiosyncrasie locale sans perdre la diversité culturelle et respecter celle-ci sans s'y arrêter $»^{26}$. épais l'écart entre les deux - entre le réalisé et l'envisagé - plutôt que de rendre compte simplement des actions menées en analysant objectivement ce qu'elles apportent aux publics. À une autre échelle, c'est ce qui arrive aux états démocratiques: ils sont systématiquement accusés de multiples déviances, graves ou minimes, par rapport au modèle de démocratie idéale. Mais cette dernière a-t-elle jamais existé ailleurs que dans les textes de philosophie politique? Évidemment, il est difficile, dans le contexte politique, économique et social actuel de ne pas céder au tout commercial, qu'il soit basé sur le ludique ou l'esthétique. Difficile aussi de ne pas céder au tout technologique, sans pour autant en revenir au caricatural musée du sabot. C'est difficile, mais tout projet militant est difficile et l'utopie de l'écomusée doit être défendue dans cet esprit, celui de la résistance à la marchandisation de la culture.

\section{BIBLIOGRAPHIE}

AUGÉ Marc (dir.), Territoires de la mémoire. Les collections du patrimoine ethnologique dans les écomusées, Thonon-les-Bains/Salins, éditions de l'Albaron, société présence du Livre/FEMS, 1992

AUGÉ Marc, « Introduction. Les paradoxes du patrimoine », dans AUGÉ Marc (dir.), Territoires de la mémoire. Les collections du patrimoine ethnologique dans les écomusées, Thonon-les-Bains/Salins, éditions de l'Albaron, société présence du Livre/FEMS, 1992, p.21

BATTESTI Jacques (dir.), Que reste-t-il du présent ? Collecter le contemporain dans les musées de société. Bordeaux/Bayonne, Le Festin/Musée basque et de l'histoire de Bayonne, 2012

BAZIN Jean, « Interpréter ou décrire. Notes critiques sur la connaissance anthropologique », Des Clous dans la Joconde, 2008, p. 407-433. Première édition dans REVEL Jacques et Nathan WACHTEL (dir.), Une École pour les sciences sociales. De la VIe section à l'École des hautes études en sciences sociales, Paris, éditions du Cerf/éditions de l'EHESS, 1996, p.401-420 
BENSA Alban, Après Lévi Strauss. Pour une anthropologie à taille humaine, Paris, Textuel, 2010

BOURDIEU Pierre, « L'objectivation participante », Actes de la Recherche en Sciences Sociales, $\mathrm{n}^{\circ} 150$, 2003, p.43-58

CHAUMIER Serge, Des musées en quête d'identité. Écomusée versus technomusée, Paris, L'Harmattan, 2003

DESCOLA Philippe, Par-delà nature et culture, Paris, Gallimard 2005

" Des rêves et de l'utopie. Carte blanche à la fédération des écomusées et musées de société ", Musée et collection publiques de France, n²43, 2006

DESVALLÉES André (dir.), «L'écomusée : rêve ou réalité », Publics et Musées, n¹7-18, 2000, https://www.persee.fr/issue/pumus_1164-5385_2000_num_17_1, consulté le 13/06/18

JOULIAN Frédéric, TASTEVIN Yann-Philippe et Jamie FURNISS (dir.), « Réparer le monde. Excès, reste et innovation ", Techniques et culture, $n^{\circ} 65-66,2016$

JOURNOT Florence et Gilles BELLAN, Archéologie de la France moderne et contemporaine, Paris, La Découverte, 2011

KARSENTI Bruno, « L'anthropologie comme science sociale », L'Homme, n² 218, 2016/2, p.253-262

LATOUR Bruno, Nous n'avons jamais été modernes, Paris, La Découverte, 1991

MAIROT Philippe, «L'objet de l'écomusée », dans AUGÉ Marc (dir.), Territoires de la mémoire. Les collections du patrimoine ethnologique dans les écomusées, Thonon-les-Bains/Salins, éditions de l'Albaron, société présence du Livre/FEMS, 1992, p.23-35

OLIVIER DE SARDAN Jean-Pierre, « La politique du terrain », Enquête, $\mathrm{n}^{\circ} 1,1995$, mis en ligne le 10 juillet 2013, https://journals.openedition.org/enquete/263, consulté le 08 novembre 2013

POULARD Frédéric, «Les écomusées. Participation des habitants et prise en compte des publics », Ethnologie française, vol. XXXVII, n³, 2007, p.551-557

RIVIÈRE Georges-Henri, « Définition évolutive de l'écomusée », Museum, Volume XXXVII, n¹48, 1985 , p.182-183

SAILLANT Francine, KILANI Monder et Florence GRAEZER-BIDEAU (dir.), Manifeste de Lausanne. Pour une anthropologie non hégémonique, Montréal, Liber, 2011

SAUTY François, Écomusées et musées de société au service du développement local, utopie ou réalité?, Lempdes, Source, Centre national de ressources du tourisme en espace rural, 2001

TORNATORE Jean Louis, « La difficile politisation du patrimoine ethnologique », Terrain, $\mathrm{n}^{\circ} 42$, 2004, p.149-160

TRAIMOND Bernard, « La critique des sources en anthropologie », Instituto de Estudios Altoaragoneses, Revista Seccion de Anthropologia Social, n¹8, 2001, p.133-157. revistas.iea.es/ index.php/AFJC/article/download/2527/2517

VARINE Hugues de, «L'écomusée, un mot, deux concepts, mille pratiques », traduction de « El ecomuseo, una palabra, dos conceptos, mil prácticas ", Mus-A, Revista de los museos de Andalucia, Sevilla, ${ }^{\circ} 8,2007$, p.19-29. http://www.hugues-devarine.eu/img/cms/

2006\%20Ecomus\%C3\%A9e.pdf, consulté le 12/06/18

VARINE Hugues de, « Musées et développement local, un bilan critique », traduction de " Museus e desenvolvimento local : um balanço crítico », dans M.C. O. Bruno, K. F. Neves (éd.), Museus como Agentes de Mudança Social e Desenvolvimento - Propostas e reflexões museológicas, Museu de 
Arqueologia de Xingo, 2008, p. 11-20. http://www.hugues-devarine.eu/img/cms/

2008\%20Mus\%C3\%A9es\%20et\%20d\%C3\%A9veloppement\%20social.pdf, consulté le 19/06/18

VARINE Hugues de, «Un musée “éclaté” : le Musée de l'homme et de l'industrie, Le Creusot-

Montceau-les-Mines », Museum, vol.XXV-4, 1973 p.242-249

\section{NOTES}

1. Voir notamment le dossier de la revue Publics et Musées, "L'écomusée : rêve ou réalité ", n¹7-18, 2000, notamment l'introduction d'André DESVALLÉES et DEBARY Octave, «L'écomusée est mort, vive le musée », op. cit., p.71-82 ; « Entretien avec Hugues de Varine », op. cit., p.203-210 ; COUSIN Saskia, «Un brin de culture, une once d'économie : écomusée et économusée », op. cit., p. 115-137 (où l'auteur parle du parcours « Du choc des utopies aux interférences du réel ») https:// www.persee.fr/issue/pumus_1164-5385_2000_num_17_1, consulté le 13/06/18. Voir aussi le dossier «Des rêves et de l'utopie. Carte blanche à la fédération des écomusées et musées de société » de la revue Musée et collection publiques de France, n²43, 2006 ou encore SAUTY François, Écomusées et musées de société au service du développement local, utopie ou réalité?, Lempdes, Source, Centre national de ressources du tourisme en espace rural, 2001.

2. MAIROT Philippe, "L'objet de l'écomusée », dans AUGÉ Marc (dir.) Territoires de la mémoire. Les collections du patrimoine ethnologique dans les écomusées, Thonon-les-Bains/Salins, éditions de l'Albaron, société présence du Livre/FEMS, 1992, p. 23-35, citation p.31.

3. VARINE Hugues de, "L'écomusée, un mot, deux concepts, mille pratiques ", Texte d'une intervention à une rencontre des musées d'Andalousie à Grenade, publié en traduction espagnole, sous le titre "El ecomuseo, una palabra, dos conceptos, mil prácticas", Mus-A, Revista de los museos de Andalucia, Sevilla, $n^{\circ} 8,2007$, p.19-29.

4. Ouvrage publié par Thomas More sous le titre latin De optimo rei publice statu, deque nova insula Utopia, http://classiques.uqac.ca/classiques/More_thomas/1_utopie/utopie_Ed_fr_1842.pdf , consulté le 15/06/18.

5. RIVIERE Georges-Henri, «Définition évolutive de l'écomusée », Museum, Volume XXXVII, n¹48, 1985, p.182-183. Ce texte est initialement daté de 1980.

6. VARINE Hugues de "L'écomusée, un mot, deux concepts... », op. cit., p.5.

7. POULARD Frédéric, «Les écomusées. Participation des habitants et prise en compte des publics ", Ethnologie française, vol. XXXVII, n³, 2007, p. 551-557; TORNATORE Jean Louis, «La difficile politisation du patrimoine ethnologique ", Terrain, $\mathrm{n}^{\circ} 42,2004, \mathrm{p} .149-160$.

8. Ce chantier coordonné par l'écomusée Creusot-Montceau et l'AFPA visait à l'insertion et la réinsertion de chômeurs longue durée ou de jeunes déscolarisés. L'opération consistait à réhabiliter une usine de production céramique en activité de 1893 à 1967, demeurée en friche et en ruine depuis sa fermeture. Le Conseil Régional subventionnait ce projet et c'est dans ce cadre que cet élu local était venu se rendre compte de l'avancée des travaux.

9. Moi-même en l'occurrence, historien de formation mais ayant pour les besoins de la cause entamé une thèse d'anthropologie sociale et ethnologie à l'EHESS (Paris).

10. En particulier contre «l'historicisme" (DESCOLA Philippe, Par-delà nature et culture, Paris, Gallimard 2005, en particulier p.15-19) et contre la sociologie (KARSENTI Bruno, «L'anthropologie comme science sociale», L'Homme, n 218, 2016/2, p.253-262. A propos d'un ouvrage de Gérard Lenclud, Bruno Karsenti remarque qu'il situe l'anthropologie par rapport à l'histoire et aux sciences naturelles, sans se référer à la sociologie).

11. BOURDIEU Pierre, "L'objectivation participante », Actes de la Recherche en Sciences Sociales, $\mathrm{n}^{\circ}$ 150, 2003, p. 43-58; citation p.57. 
12. JOURNOT Florence et Gilles BELLAN, Archéologie de la France moderne et contemporaine, Paris, La Découverte, 2011, p.162.

13. OLIVIER DE SARDAN Jean-Pierre, 1995 « La politique du terrain », Enquête [En ligne], n¹, mis en ligne le 10 juillet 2013, https://journals.openedition.org/enquete/263, consulté le 08 novembre 2013, citation p.4, $\$ 11$.

14. BAZIN Jean, «Interpréter ou décrire. Notes critiques sur la connaissance anthropologique », Des Clous dans la Joconde, 2008, p. 409. Première édition dans REVEL Jacques et Nathan WACHTEL (dir.), Une École pour les sciences sociales. De la VI ection à l'École des hautes études en sciences sociales, Paris, éditions du Cerf/éditions de l'EHESS, 1996, p.401-420.

15. C'est ce qu'écrivait Hugues de Varine au sujet du «musée éclaté » du Creusot en 1973 : «Le musée n'a pas de visiteurs, il a des habitants ». DE VARINE Hugues, "Un musée "éclaté": le Musée de l'homme et de l'industrie, Le Creusot-Montceau-les-Mines », Museum, vol. XXV-4, 1973 p.242-249, citation p.244.

16. POULARD Frédéric, op. cit., p. 554. Selon lui, le contrôle de la programmation culturelle reste l'apanage des professionnels, même si la population est invitée à s'exprimer. Voir aussi CHAUMIER Serge, Des musées en quête d'identité. Ecomusée versus technomusée, Paris, L'Harmattan, 2003.

17. SAILLANT Francine, KILANI Monder et Florence GRAEZER-BIDEAU (dir.) Manifeste de Lausanne. Pour une anthropologie non hégémonique, Montréal, Liber, 2011, citation p.18.

18. TRAIMOND Bernard "La critique des sources en anthropologie», Instituto de Estudios Altoaragoneses, Revista Seccion de Anthropologia Social, 2001, p.133-157, citation p.133.

19. BENSA Alban, Après Lévi Strauss. Pour une anthropologie à taille humaine, Paris, Textuel, 2010, p. 39.

20. OLIVIER DE SARDAN Jean-Pierre, op. cit., citation p.4, $\$ 11$.

21. LATOUR Bruno, Nous n'avons jamais été modernes, Paris, La Découverte 1991 et DESCOLA Philippe, op. cit.

22. Je renvoie aux travaux de Daniel Fabre, Nathalie Heinich, Michel Rautenberg, Jean-Louis Tornatore, entre autres.

23. On pense à l'écomusée du Val de Bièvre ou au Musée Dauphinois notamment. Pour un panorama, voir BATTESTI Jacques (dir.) Que reste-t-il du présent? Collecter le contemporain dans les musées de société. Bordeaux/Bayonne, Le Festin/Musée basque et de l'histoire de Bayonne, 2012.

24. Voir par exemple JOULIAN Frédéric, TASTEVIN Yann-Philippe et Jamie FURNISS (dir.), « Réparer le monde. Excès, reste et innovation », Techniques et culture, n65-66, 2016.

25. VARINE Hugues de, "Musées et développement local, un bilan critique " 2008. Traduction d'un article publié, en portugais, dans "Museus como Agentes de Mudança Social e Desenvolvimento - Propostas e reflexões museológicas", une publication du Museu de Arqueologia de Xingó (M.C. O. Bruno, K. F. Neves, ed.), 2008, p.11-20.

26. AUGÉ Marc, «Introduction. Les paradoxes du patrimoine », dans AUGÉ Marc (dir.), op. cit., p. 21.

\section{RÉSUMÉS}

Les écomusées ont été créés au début des années 1970 sur la base d'un ensemble d'idées utopiques : musée sans collection, sans visiteurs mais avec des habitants, miroir tendu vers soi- 
même et vers l'Autre. A posteriori, les analystes ont souvent fait de cette utopie une aporie, une impasse logique. La recherche en sciences sociales, l'ethnologie principalement, était le moteur de ces institutions théoriquement participatives. Cet article propose de se réapproprier l'utopie initiale en pratiquant une recherche anthropologique critique et réflexive, permettant aux publics de participer à la construction des savoirs et au développement culturel et social par l'action écomuséale.

Ecomuseums were created in the early 1970s on the basis of a set of utopian ideas: museum without collection, without visitors but with inhabitants, a mirror offered to oneself and to the Other. A posteriori, analysts have often turned this utopia into an aporia, a logical impasse. Social science research, mainly ethnology, was the driving force behind these institutions, theoretically participative. This article wants to appropriate the initial utopia by driving a critical and reflexive anthropological research, allowing the publics to produce knowledge and to build cultural and social development through ecomuseal action.

\section{INDEX}

Mots-clés : histoire des techniques, utopie, musée, écomusée, patrimoine, patrimoine culturel, recherche

Keywords : history of technology, utopia, museum, eco-museum, heritage, cultural heritage, research

\section{AUTEUR}

\section{THIERRY BONNOT}

Thierry Bonnot est anthropologue, chargé de recherche au CNRS, membre de l'Institut de Recherche Interdisciplinaire sur les Enjeux Sociaux (IRIS, Paris). Ses recherches portent essentiellement sur le statut social des objets, leurs modes d'appropriation et la constitution des patrimoines, dans une optique pluridisciplinaire (anthropologie, histoire, archéologie). Il a travaillé une dizaine d'années à l'écomusée Le Creusot-Montceau et continue de participer à ses activités. Dernier ouvrage paru : L'attachement aux choses, Paris, éditions du CNRS, 2014. 